\title{
Fractal geometry model for effective thermal conductivity of three-phase porous media
}

\author{
Yongting $\mathrm{Ma}$ \\ Department of Physics and the State Key Laboratory of Leser Technology, Huazhong University of Science \\ and Technology, 1037 Luoyu Road, Wuhan 430074, People's Republic of China \\ Boming $\mathrm{Yu}^{\mathrm{a})}$ \\ Department of Physics and the State Key Laboratory of Leser Technology, Huazhong University of Science \\ and Technology, 1037 Luoyu Road, Wuhan 430074, People's Republic of China; The State Key \\ Laboratory of Nonlinear Mechanics (LNM), Institute of Mechanics, Chinese Academy of Science, Beijing, \\ 100080, Peoples Republic of China \\ Duanming Zhang \\ Department of Physics and the State Key Laboratory of Leser Technology, Huazhong University of Science \\ and Technology, 1037 Luoyu Road, Wuhan 430074, People's Republic of China \\ Mingqing Zou \\ Department of Physics, and the State Key Laboratory of Leser Technology, Huazhong University of Science \\ and Technology, 1037 Luoyu Road, Wuhan A30074, People's Republic of China
}

(Received 18 September 2003; accepted 23 February 2004)

\begin{abstract}
An approximate model, a fractal geometry model, for the effective thermal conductivity of three-phase/unsaturated porous media is proposed based on the thermal-electrical analogy technique and on statistical self-similarity of porous media. The proposed thermal conductivity model is expressed as a function of porosity (related to stage $n$ of Sierpínski carpet), ratio of areas, ratio of component thermal conductivities, and saturation. The recursive algorithm for the thermal conductivity by the proposed model is presented and found to be quite simple. The model predictions are compared with the existing measurements. Good agreement is found between the present model predictions and the existing experimental data. This verifies the validity of the proposed model. (C) 2004 American Institute of Physics. [DOI: 10.1063/1.1703820]
\end{abstract}

\section{INTRODUCTION}

The effective thermal conductivity of three-phase/ unsaturated porous media, such as soil, oil-filled porous rocks, concrete, fiber reinforced composites, etc., has been a focus of studies of properties of these media. In the past, many models for the effective thermal conductivity of the media were proposed.

Nozad et al. ${ }^{1}$ used a transient method to determine the effective thermal conductivity for three-phase systems, however, the method of volume averaging and the closure scheme increased the computational difficulties and they were not able to explore three-phase systems as thoroughly as the two-phase systems. Theoretical predictions of the thermal conductivity for three-phase systems were found to be $33 \%$ lower than the measured values.

Singh $e t$ al. ${ }^{2}$ conducted an experimental investigation by using dune sand and brick sand samples at porosities of 0.42 and 0.52 , respectively, to determine the thermal conductivity of three-phase/unsaturated porous media. The moisture content in dune sand (moistened with distilled water) was about $25 \%$ by weight. When the sample was saturated with tertiary amyl alcohol, the content of alcohol was $23.3 \%$ by weight. In the case of brick sand, the content of water observed was

a) Author to whom the correspondence should be addressed; electronic mail: yu3838@public.wh.hb.cn; m_yt@163.com
$47.5 \%$ by weight. Determination of moisture content was made by weighing a specimen sample before and after drying. The constant temperature of the sample is maintained by liquid circulating at the required temperature around the soil sample. A probe is placed along the central axis of a cylindrical soil container. The ETC (effective thermal conductivity) of the system is calculated by the relation $\lambda_{\exp }$ $=Q \ln \left(t_{2} / t_{1}\right) /\left[4 \pi\left(T_{2}-T_{1}\right)\right]$, where $T_{2}$ and $T_{1}$ are the temperatures at times $t_{2}$ and $t_{1}$ respectively. By knowing the power $Q$ per unit length supplied to the probe heater, the ETC of the sample can be determined. It appears that the ETC determined by the experiments of Singh et al. was based on the one-dimensional heat conduction, and the two-dimensional samples were used in their experiments.

$\mathrm{Hu}$ et $\mathrm{al}^{3}{ }^{3}$ derived a model for the thermal conductivity of unconsolidated porous medium. Their model is based on capillary pressure-saturation relation and also has two empirical constants with no physical meaning. Abu-Hamdeh et $a .^{4}$ applied two methods, the hot wire method and the heating-cooling method, to evaluate thermal conductivity of some soils, and they studied the effect of bulk density and moisture content on thermal conductivity. The results were correlated as two correlations, also with several empirical constants. Gori and Corasaniti ${ }^{5}$ presented a theoretical model for the effective thermal conductivity of unsaturated soils at moderately high temperatures by taking into account the special nature of the soil. Their model assumes a unit cell to be 
composed of a cube with a cubic solid particle at the center. The thermal conductivity is evaluated by solving the heat conduction equation with the assumption of parallel isotherms within the cubic space (i.e., one-dimensional heat flow assumption). The results were correlated according to four regions, each with the different constants obtained by fitting their experimental data.

Many researchers ${ }^{6-11}$ have proved that porous media in nature are fractals statistically, meaning that they are statistically self-similar. The statistical self-similarity of porous media suggests that the fractal theory and technique may be used to predict properties of a porous medium. Thovert et al. $^{7}$ and Adler ${ }^{9}$ studied and reviewed the thermal conductivity for both random and deterministic fractals, and they applied the Sierpínski carpet as a model to simulate real porous media. The dimensionless thermal conductivity was correlated as Archie's law with two empirical constants.

Recently, Richter and Viljoen ${ }^{12}$ calculated the effective thermal conductivity of particulate mixtures by constructing a probability distribution for contacts based on the bijective mapping of discrete points on surfaces of species onto each other and by considering the progression problem as the probability that a phonon could progress from one layer to the next. However, this model is limited to two-species/ phases, so it is obvious that additional species will introduce considerably more complexity in the problem. Another weakness in the model is that the number of layers is not analytically related to the volume fraction of the species.

Most recently, Ma et al. ${ }^{13}$ used the Sierpínski carpet as a geometry model and developed a self-similarity model to simulate the statistically self-similar porous medium to predict the thermal conductivity of two-phase/saturated porous media based on the thermal-electrical analogy technique and one-dimensional heat flow assumption. For detailed justification of the one-dimensional heat flow assumption, readers may consult the work by Ma et al. ${ }^{13}$

This work extends our previous model, ${ }^{13}$ a self-similar model for the effective thermal conductivity of two-phase/ saturated porous media, to that for three-phase or unsaturated porous media based on the fact that the porous media both saturated and unsaturated are fractals and can be described by statistical self-similarity existing in the media. For this purpose, the Sierpínski carpet geometry model is again chosen to approximate the statistically self-similar unsaturated porous media. Then, the thermal-electrical analogy technique is applied and the effective thermal conductivity for statistically self-similar unsaturated porous media is derived. Finally, the model predictions are compared with the existing experimental results.

\section{BASIC CHARACTERS OF FRACTAL MEDIA}

Euclidean geometry describes ordered objects such as points, curves, surfaces, and cubes using integer dimension $0,1,2$, and 3 , respectively. The measure of an object such as the length of a line, the area of a surface, and the volume of a cube is related to its dimension and is invariant with respect to the unit of measurement used. However, numerous objects found in nature ${ }^{14}$ such as rough surfaces, coastlines,

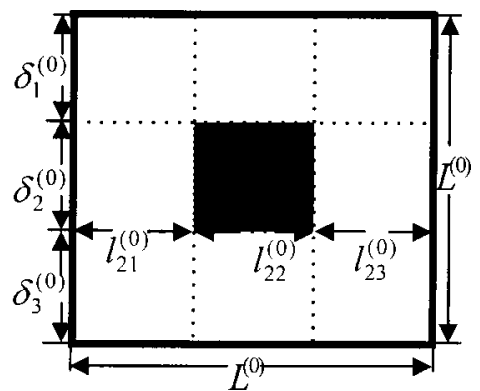

(a)

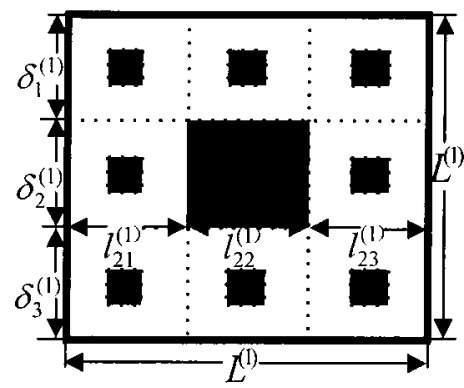

(b)

FIG. 1. Sierpínski carpets and division of regions (a) 0-stage Sierpínski carpet and (b) 1-stage Sierpínski carpet.

mountains, rivers, lacks, and islands, are disordered and irregular, and they do not follow the Euclidean description due to the scale-dependent measures of length, area, and volume. These objects are called fractals, and the dimensions of such objects are nonintegral and defined as fractal dimensions. The measure of a fractal object $M(L)$ is related to the length scale $L$ through a scaling law in the form of ${ }^{14}$

$$
M(L) \sim L^{D_{f}},
$$

where $M$ can be the length of a line or the area of a surface or the volume of a cube or the mass of an object, and $D_{f}$ is the fractal dimension of an object. The sign " " can be replaced by "=" for exactly self-similar fractals such as $\mathrm{Si}$ erpínski carpet and Sierpínski gasket ${ }^{14}$ and "minimal overlap" self-similar fractals. ${ }^{15}$ There are two kinds of fractal geometry in self-similar sets: one is exactly self-similar fractals such as Sierpínski carpet, ${ }^{14}$ see Fig. 1, and the other is "minimal overlap" self-similar fractals, ${ }^{15}$ see Fig. 2. Figure 1 displays the exactly self-similar fractals, 0 -stage [Fig. 1(a)] and 1-stage [Fig. 1(b)] Sierpínski carpets. The 0-stage carpet or generator can be built by cutting out or filling the central part in a square. The 1-stage carpet can be obtained by translation and dilation of a 0 -stage carpet, and so on. The ratio between the sides of successively sized square is 3 . Figure 2 shows the Sierpínski carpets with "minimal overlap" to approximate the three-phase/unsaturated porous media. Equation (1) implies the property of self-similarity, which means that the value of $D_{f}$ from Eq. (1) is a constant over a certain range of length scales $L$. The geometry structures such as Sierpínski carpet, Sierpínski gasket, and Koch curve are the examples of the exactly self-similar fractals, which exhibit the self-similarity over an infinite range of length scales. ${ }^{14,16}$ However, exactly self-similar fractals in a global range are 


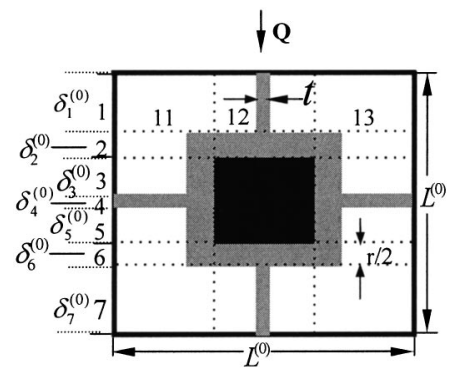

(a)

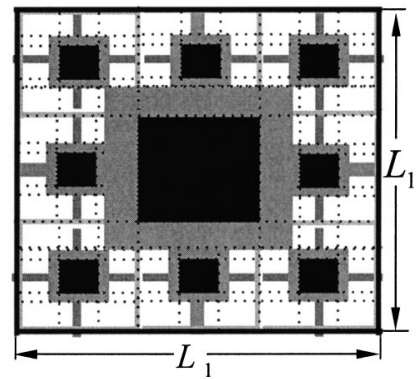

(c)

FIG. 2. The thermal conductivity model and the thermal-electrical analogy for a 0 -stage and 1-stage carpet in three phases (a) the thermal conductivity model for 0-stage carpet, (b) the network of the thermal-electrical analogy for 0-stage carpet, and (c) the thermal conductivity model for 1-stage carpet.

rarely found in nature. Many objects found in nature are not exactly self-similar, they are statistically self-similar, such as real porous media. For a real porous medium, the microstructure such as pore sizes and particles are not exactly selfsimilar, and they are statistically self-similar. This medium can be called the fractal porous medium.

\section{EFFECTIVE THERMAL CONDUCTIVITY FOR THREE-PHASE/UNSATURATED FRACTAL POROUS MEDIA}

It is known that heat conduction through porous media strongly depends on the thermal conductivity ratio of components of the medium, and on porosity and saturation. To analyze the thermal conductivity of a porous medium, it is usually assumed that the medium has a periodic structure, and a unit cell or representative cell is then applied. Usually there are three approaches, Fourier's Law models, Ohm's Law models, and empirical models, to predict the thermal conductivity. Crane and Vachon ${ }^{17}$ compared the two approaches, Fourier's Law models and Ohm's Law models, for prediction of the thermal conductivity. Ma et al. ${ }^{13}$ made a brief discussion on the three approaches and applied the Ohm's Law model to develop a self-similarity model for effective thermal conductivity of saturated porous media. The present work continues to apply the Ohm's Law model approach and to extend our previous work to develop a fractal geometry model for effective thermal conductivity of three-phase/unsaturated porous media.

It is also assumed that the unsaturated porous medium consists of two portions, randomly distributed nontouching particles surrounded by the wetting phase and self-similarly distributed particles also surrounded by the wetting phase contacting each other with the thermal resistance formed by the wetting phase. The latter is simulated by Sierpínski carpet, a self-similar fractal geometry model. Then the thermalelectrical analogy is used for thermal conductivity.

Figure 1 gives the exactly self-similar fractals, Sierpínski carpets, to model the statistically self-similar fractal porous media. The whites in Fig. 1 can be considered pores and the black are solid particles. The fractal dimension for Sierpínski carpet as shown in Fig. 1 is $D_{f}=\ln 8 / \ln 3=1.893 .{ }^{14}$ The porosity of the Sierpínski carpet $\varphi$ is given by

$$
\varphi=(8 / 9)^{n+1},
$$

where the superscript $n$ represents the stage of Sierpínski carpet, and $n=0,1,2, \ldots$. The heat conduction model for the three-phase porous media is shown in Fig. 2. In Fig. 2, the black represents the particles, the gray is the wetting phase (e.g., water), and the white is nonwetting phase (e.g., gas). Figure 2(a) is a 0-stage Sierpínski carpet, Fig. 2(b) is the network of resistance of Fig. 2(a). Figure 2(c) is a 1-stage Sierpínski carpet, which is a "minimal overlap" fractal. ${ }^{15}$ The network of resistance Fig. 2(c) can be drawn by the same way as Fig. 2(b). The bars represent the contact resistance between particles. So, the wetting phase includes the wetting phase content $S_{\text {wsp }}$ (the subscript wsp represents the wetting phase thickness of surrounding particles) surrounding the particles, and the barrier content $S_{\mathrm{wb}}$ (the subscript wb refers to the wetting phase width of barrier) of the wetting phase.

The saturation is defined by $S_{w}=V_{w} / V_{p}$ (here $V$ is the volume, the subscripts $w$ and $p$ represent the wetting phase and pore, respectively), which satisfies

$$
S_{w}^{(0)}=S_{\mathrm{wsp}}^{(0)}+S_{\mathrm{wb}}^{(0)},
$$

where the superscript 0 represents the 0-stage Sierpínski carpet.

For the 0-stage carpet, the wetting phase content $S_{\text {wsp }}^{(0)}$ surrounding the particles is expressed as

$$
S_{\mathrm{wsp}}^{(0)}=\frac{9}{8}\left[\left(\frac{1}{3}+r^{+}\right)^{2}-\left(\frac{1}{3}\right)^{2}\right] .
$$

See Appendix A for derivation of the wetting phase content $S_{\text {wsp }}^{(0)}$ surrounding the particles.

Because of self-similarity, for the $n$-stage carpet, the wetting phase content $S_{\mathrm{wsp}}^{(n)}$ of surrounding the particles can be obtained by

$$
\begin{gathered}
S_{\mathrm{wsp}}^{(n)}=9\left[\left(\frac{9}{8}\right)^{n+1}-1\right]\left[\left(\frac{1}{3}+r^{+}\right)^{2}-\left(\frac{1}{3}\right)^{2}\right] \\
(n=0,1,2, \ldots,) .
\end{gathered}
$$

See Appendix A for derivation.

For the 0-stage carpet, the barrier content $S_{\mathrm{wb}}^{(0)}$ of wetting phase is derived as

$$
S_{\mathrm{wb}}^{(0)}=\frac{9}{8} \times 4 t^{+}\left(\frac{1}{3}-\frac{r^{+}}{2}\right) .
$$

See Appendix B for derivation. 
Similarly, because of self-similarity, for the $n$-stage carpet, the barrier content $S_{\mathrm{wb}}^{(n)}$ of wetting phase can be obtained by

$$
S_{\mathrm{wb}}^{(n)}=9\left[\left(\frac{9}{8}\right)^{n+1}-1\right] \times 4 t^{+}\left(\frac{1}{3}-\frac{r^{+}}{2}\right) \quad(n=0,1,2, \ldots,) .
$$

See Appendix B for derivation.

So, if a value of porosity $\varphi$ is given, according to Eq. (2), the stage $n$ of a Sierpínski carpet can be found. If the values of $S_{w}$ and $S_{\mathrm{wsp}} / S_{w}$ are known, the parameters $r^{+}$and $t^{+}$can be found from Eqs. (3), (4), and (5). In this model, $S_{\mathrm{wsp}} / S_{w}=0.8$ is used, the same value as that in Ref. 5 .

In this work, the one-dimensional heat flow is again assumed, the same as our previous work. ${ }^{13}$ Figure 2(b) displays the series-parallel network for the electrical analogy of the thermal resistances of the 0 -stage Sierpínski carpet. It is expected that the contact thermal resistance between particles depends on the bar width $t$. In this simulation, $t=0$ means perfect contact between particles. Since the Sierpínski carpet is a self-similar fractal geometry, the contact thermal resistances or bar widths and the thickness of wetting phase surrounding the particle are also self-similar but might be "minimal overlap" fractals, ${ }^{15}$ as interpreted in Sec. II.

The total resistance of 0-stage Sierpínski carpet is

$$
\begin{aligned}
R_{\mathrm{sc}}^{(0)}= & \frac{1}{k_{g}}\left\{\frac{\delta_{1}^{(0)}+\delta_{7}^{(0)}}{L^{(0)}\left[t^{+} \beta_{\mathrm{wg}}^{(0)}+\left(1-t^{+}\right)\right]}\right. \\
& +\frac{\delta_{2}^{(0)}+\delta_{6}^{(0)}}{\left(l_{22}^{(0)}+r\right) \beta_{\mathrm{wg}}^{(0)}+l_{21}^{(0)}+l_{23}^{(0)}-r} \\
& +\frac{\delta_{3}^{(0)}+\delta_{5}^{(0)}}{l_{31}^{(0)}+l_{33}^{(0)}-r+\beta_{\mathrm{wg}}^{(0)} r+\beta_{\mathrm{sg}}^{(0)} l_{32}^{(0)}} \\
& \left.+\frac{\delta_{4}^{(0)}}{\left(l_{41}^{(0)}+l_{43}^{(0)}\right) \beta_{\mathrm{wg}}^{(0)}+\beta_{\mathrm{sg}}^{(0)} l_{42}^{(0)}}\right\} .
\end{aligned}
$$

See Appendix $\mathrm{C}$ for derivation of the total resistance of a 0 -stage Sierpínski carpet. Thus, the dimensionless effective thermal conductivity for the 0 -stage carpet can be obtained by

$$
\begin{aligned}
k_{e, \mathrm{sc}}^{+(0)}= & \frac{k_{e, \mathrm{sc}}^{(0)}}{k_{g}}=\frac{L^{(0)}}{R_{\mathrm{sc}}^{(0)} A^{(0)} k_{g}}=\left\{\frac{\delta_{1}^{(0)}+\delta_{7}^{(0)}}{L^{(0)}\left[t^{+} \beta_{\mathrm{wg}}^{(0)}+\left(1-t^{+}\right)\right]}\right. \\
& +\frac{\delta_{2}^{(0)}+\delta_{6}^{(0)}}{\left(l_{22}^{(0)}+r\right) \beta_{\mathrm{wg}}^{(0)}+l_{21}^{(0)}+l_{23}^{(0)}-r} \\
& +\frac{\delta_{3}^{(0)}+\delta_{5}^{(0)}}{l_{31}^{(0)}+l_{33}^{(0)}-r+\beta_{\mathrm{wg}}^{(0)} r+\beta_{\mathrm{sg}}^{(0)} l_{32}^{(0)}} \\
& \left.+\frac{\delta_{4}^{(0)}}{\left(l_{41}^{(0)}+l_{43}^{(0)}\right) \beta_{\mathrm{wg}}^{(0)}+\beta_{\mathrm{sg}}^{(0)} l_{42}^{(0)}}\right\}^{-1},
\end{aligned}
$$

where $A^{(0)}=L^{(0)} \times 1, A$ is the total area of a representative cross section, $R$ is resistance, the subscript sc represents Sierpínski carpet, $L$ is the side length of Sierpínski carpet, $\beta_{\mathrm{wg}}=k_{w} / k_{g}$ is the ratio of thermal conductivities $k_{w}$ (wetting phase) to $k_{g}$ (nonwetting phase), $\beta_{\text {sg }}=k_{s} / k_{g}$ is the ratio of thermal conductivities $k_{s}$ (solid phase) to $k_{g}$ (nonwetting phase), $\delta$ =thickness of layer, and $l$ is the geometry length, see Fig. 1 for definition of all geometry lengths.

The similar method can be used to derive the effective thermal conductivity of a 1-stage carpet, except for the central shade with the conductivity $k_{s}$ (the subscript $s$ represents the solid phase), the other regions are considered as an equivalent homogeneous material with the thermal conductivity $k_{e, s c}^{(0)}$. Thus, the dimensionless effective thermal conductivity of the 1-stage carpet is

$$
\begin{aligned}
k_{e, \mathrm{sc}}^{+(1)}= & k_{e, \mathrm{sc}}^{+(0)}\left\{\frac{\delta_{1}^{(1)}+\delta_{7}^{(1)}}{L^{(1)}\left[t^{+} \beta_{\mathrm{wg}}^{(1)}+\left(1-t^{+}\right)\right]}\right. \\
& +\frac{\delta_{2}^{(1)}+\delta_{6}^{(1)}}{\left(l_{22}^{(1)}+r\right) \beta_{\mathrm{wg}}^{(1)}+l_{21}^{(1)}+l_{23}^{(1)}-r} \\
& +\frac{\delta_{3}^{(1)}+\delta_{5}^{(1)}}{l_{31}^{(1)}+l_{33}^{(1)}-r+\beta_{\mathrm{wg}}^{(1)} r+\beta_{\mathrm{sg}}^{(1)} l_{32}^{(1)}} \\
& \left.+\frac{\delta_{4}^{(1)}}{\left(l_{41}^{(1)}+l_{43}^{(1)}\right) \beta_{\mathrm{wg}}^{(1)}+\beta_{\mathrm{sg}}^{(1)} l_{42}^{(1)}}\right\}^{-1},
\end{aligned}
$$

where $\beta_{\mathrm{wg}}^{(1)}=\beta_{\mathrm{wg}}^{(0)} / k_{e, \mathrm{sc}}^{+(0)}, \beta_{\mathrm{sg}}^{(1)}=\beta_{\mathrm{sg}}^{(0)} / k_{e, \mathrm{sc}}^{+(0)}$.

For a $n$-stage carpet, the dimensionless effective thermal conductivity is

$$
\begin{aligned}
k_{e, \mathrm{sc}}^{+(n)}= & k_{e, \mathrm{sc}}^{+(n-1)}\left\{\frac{\delta_{1}^{(n)}+\delta_{7}^{(n)}}{L^{(n)}\left[t^{+} \beta_{\mathrm{wg}}^{(n)}+\left(1-t^{+}\right)\right]}\right. \\
& +\frac{\delta_{2}^{(n)}+\delta_{6}^{(n)}}{\left(l_{22}^{(n)}+r\right) \beta_{\mathrm{wg}}^{(n)}+l_{21}^{(n)}+l_{23}^{(n)}-r} \\
& +\frac{\delta_{3}^{(n)}+\delta_{5}^{(n)}}{l_{31}^{(n)}+l_{33}^{(n)}-r+\beta_{\mathrm{wg}}^{(n)} r+\beta_{\mathrm{sg}}^{(n)}} l_{32}^{(n)} \\
& \left.+\frac{\delta_{4}^{(n)}}{\left(l_{41}^{(n)}+l_{43}^{(n)}\right) \beta_{\mathrm{wg}}^{(n)}+\beta_{\mathrm{sg}}^{(n)} l_{42}^{(n)}}\right\}^{-1},
\end{aligned}
$$

where $\beta_{\mathrm{wg}}^{(n)}=\beta_{\mathrm{wg}}^{(0)} / k_{e, \mathrm{sc}}^{+(n-1)}$ and $\beta_{\mathrm{sg}}^{(n)}=\beta_{\mathrm{sg}}^{(0)} / k_{e, \mathrm{sc}}^{+(n-1)}$.

$$
\begin{aligned}
& \text { Because of self-similarity, } \frac{\delta_{1}^{(n)}+\delta_{2}^{(n)}}{L^{(n)}} \\
& =\frac{\delta_{3}^{(n)}+\delta_{4}^{(n)}+\delta_{5}^{(n)}}{L^{(n)}}=\frac{\delta_{6}^{(n)}+\delta_{7}^{(n)}}{L^{(n)}}=\frac{1}{3}, \\
& \text { and } \frac{\delta_{1}^{(n)}+\delta_{7}^{(n)}}{L^{(n)}}=\frac{2}{3}-r^{+}, \frac{\delta_{2}^{(n)}+\delta_{6}^{(n)}}{L^{(n)}}=r^{+},
\end{aligned}
$$

$\frac{\delta_{3}^{(n)}+\delta_{5}^{(n)}}{L^{(n)}}=\frac{1}{3}-t^{+}$and $\frac{\delta_{4}^{(n)}}{L^{(n)}}=t^{+}$

Eq. (9a) can thus be reduced to 


$$
\begin{aligned}
k_{e, \mathrm{sc}}^{+(n)}= & k_{e, \mathrm{sc}}^{+(n-1)}\left\{\frac{2 / 3-r^{+}}{\left[t^{+} \beta_{\mathrm{wg}}^{(n)}+\left(1-t^{+}\right)\right]}\right. \\
& +\frac{r^{+}}{\left(1 / 3+r^{+}\right) \beta_{\mathrm{wg}}^{(n)}+\left(2 / 3-r^{+}\right)} \\
& +\frac{1 / 3-t^{+}}{2 / 3-r^{+}+\beta_{\mathrm{wg}}^{(n)} r^{+}+\beta_{\mathrm{sg}}^{(n)} / 3} \\
& \left.+\frac{t^{+}}{2 \beta_{\mathrm{wg}}^{(n)} / 3+\beta_{\mathrm{sg}}^{(n)} / 3}\right\}^{-1},
\end{aligned}
$$

where $\beta_{\mathrm{wg}}^{(n)}=\beta_{\mathrm{wg}}^{(0)} / k_{e, \mathrm{sc}}^{+(n-1)}$ and $\beta_{\mathrm{sg}}^{(n)}=\beta_{\mathrm{sg}}^{(0)} / k_{e, \mathrm{sc}}^{+(n-1)}$, the superscript $n=1,2, \ldots$. Equation (10) presents the recursive algorithm for thermal conductivity of self-similarly unsaturated or three-phase porous media. The calculation of the thermal conductivity is simple, for example, the thermal conductivity $k_{e, \mathrm{sc}}^{+(0)}$ of the 0 -stage carpet is calculated from Eq. (7), while the thermal conductivity of the 1-stage carpet $k_{e \text {,sc }}^{+(1)}$ can be obtained by Eq. (10) with $\beta_{\mathrm{wg}}^{(1)}=\beta_{\mathrm{wg}}^{(0)} / k_{e \text {,sc }}^{+(0)}$ and $\beta_{\mathrm{sg}}^{(1)}=\beta_{\mathrm{sg}}^{(0)} / k_{e, \mathrm{sc}}^{+(0)}$. After $k_{e, \mathrm{sc}}^{+(1)}$ is obtained, the thermal conductivity $k_{e, \mathrm{sc}}^{+(2)}$ of the 2-stage carpet can be found by again using Eq. (10) with $\beta_{\mathrm{wg}}^{(2)}=\beta_{\mathrm{wg}}^{(0)} / k_{e \text {, sc }}^{+(1)}$ and $\beta_{\mathrm{sg}}^{(2)}=\beta_{\mathrm{sg}}^{(0)} / k_{e, \mathrm{sc}}^{+(1)}$, and so forth. It is evident that the algorithm for the thermal conductivity is quite simple.

It may be recalled that the present model assumes that the porous media consists of two portions: some particles contact each other, whereas others do not touch each other (nontouching). These two portions are considered to be in parallel. So, the total thermal resistance can be expressed as

$$
1 / R_{t}=1 / R_{\mathrm{nt}}+1 / R_{\mathrm{sc}},
$$

where the subscript nt refers to the nontouching particle.

The effective thermal conductivity of the system can be obtained by

$$
\begin{aligned}
k_{e} & =\frac{1}{R_{t}} \frac{L}{A}=\frac{1}{R_{\mathrm{nt}}} \frac{L}{A}+\frac{1}{R_{\mathrm{sc}}} \frac{L}{A}=\frac{1}{R_{\mathrm{nt}}} \frac{L}{A_{\mathrm{nt}}} \frac{A_{\mathrm{nt}}}{A}+\frac{1}{R_{\mathrm{sc}}} \frac{L}{A_{\mathrm{sc}}} \frac{A_{\mathrm{sc}}}{A} \\
& =\frac{A_{\mathrm{nt}}}{A} k_{e, \mathrm{nt}}+\left(1-\frac{A_{\mathrm{nt}}}{A}\right) k_{e, \mathrm{sc}},
\end{aligned}
$$

where $A$ is the total area of a representative cross section and $A_{\mathrm{nt}}$ is an equivalent area of a cross section having the same porosity as the nontouching particles, with $0 \leqslant A_{\mathrm{nt}} / A \leqslant 1$ and $A_{\mathrm{sc}} / A=1-A_{\mathrm{nt}} / A$.

The dimensionless thermal conductivity for the nontouching particle portion is

$$
\begin{aligned}
k_{e, \mathrm{nt}}^{+}= & \frac{l_{t}}{R_{t} A_{t} k_{g}}=\left\{\frac{B-1-F / 2}{B}+\frac{F}{2\left[(B-1)+\beta_{\mathrm{wg}}\right]}\right. \\
& \left.+\frac{1}{\beta_{\mathrm{sg}}+F \beta_{\mathrm{wg}} / 2+(B-1-F / 2)}\right\}^{-1},
\end{aligned}
$$

where $A_{t}=l_{t} \times 1, \beta_{\mathrm{wg}}=k_{w} / k_{g}$ and $\beta_{\mathrm{sg}}=k_{s} / k_{g}$, see Fig. 3 and Appendix D for derivation.

Combining Eqs. (10), (12), and (13) results in the effective thermal conductivity expression in the dimensionless form as (a)

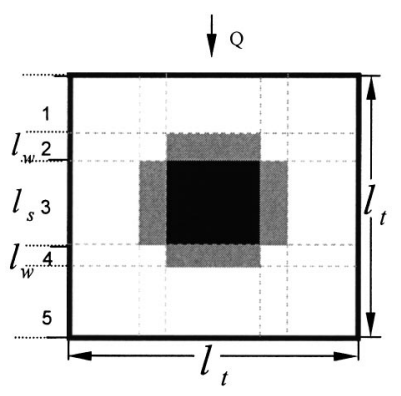

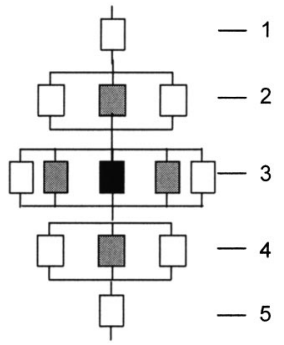

(b)
FIG. 3. The thermal conductivity model and the thermal-electrical analogy for nontouching particle in three phases (a) a unit cell for nontouching particle and (b) the network of the thermal-electrical analogy.

$$
\begin{aligned}
k_{e}^{+} & =\frac{k_{e}}{k_{g}}=\frac{A_{\mathrm{nt}}}{A} k_{e, \mathrm{nt}}^{+}+\left(1-\frac{A_{\mathrm{nt}}}{A}\right) k_{e, \mathrm{sc}}^{+} \\
& =\frac{A_{\mathrm{nt}}}{A} k_{e, \mathrm{nt}}^{+}+\left(1-\frac{A_{\mathrm{nt}}}{A}\right) k_{e, \mathrm{sc}}^{+(n)},
\end{aligned}
$$

where superscript $n=1,2,3, \ldots, k_{e, \mathrm{nt}}^{+}=k_{e, \mathrm{nt}} / k_{g}$ is given by Eq. (13), and $k_{e, \mathrm{sc}}^{+(n)}$ is computed by Eq. (10). The recursive algorithm for the effective thermal conductivity is summarized as follows:

(a) Set the initial values of $\beta_{\mathrm{wg}}^{(0)}\left(=k_{w} / k_{g}\right), \quad \beta_{\mathrm{sg}}^{(0)}$ $\left(=k_{s} / k_{g}\right)$, porosity $\varphi$, saturation $S_{w}$, area ratio $A_{\mathrm{nt}} / A$ $(=0.55)$ and ratio of $S_{\mathrm{wsp}} / S_{w}(=0.8)$, find contact parameter $t^{+}$, wetting phase parameter $r^{+}$from Eqs. (3)(5);

(b) Calculate the effective thermal conductivity $k_{e, s c}^{+(0)}$ of a 0-stage carpet from Eq. (7);

(c) Calculate the values of $\beta_{\mathrm{wg}}^{(n)}\left(=\beta_{\mathrm{wg}}^{(0)} / k_{e, \mathrm{sc}}^{+(n-1)}, n\right.$ $=1,2,3, \ldots$,$) and \beta_{\mathrm{sg}}^{(n)}\left(=\beta_{\mathrm{sg}}^{(0)} / k_{e, \mathrm{sc}}^{+(n-1)}, n=1,2,3, \ldots,\right)$;

(d) Find the effective thermal conductivity $k_{e, s c}^{+(n)}$ from Eq. (10) for the self-similar porous medium and $k_{e, \mathrm{nt}}^{+(n)}$ from Eq. (13) for the nontouching particle portion;

(e) Find the effective thermal conductivity from Eq. (14).

The above procedures $c$ and $d$ are repeated until the required Sierpínski carpet's stage $n$ (say, $n=7$ ) for the effective thermal conductivity of the self-similar porous medium. Once the thermal conductivities for both the nontouching particle portion and the self-similar portion are obtained, the effective thermal conductivity of the whole medium can be found from procedure $e$. It is seen that the present algorithm for the thermal conductivity is quite simple, and it takes less than one second in a microcomputer for one configuration (at a given porosity). Moreover, the present model has the following advantages over the other models and numerical methods:

(a) In the present model, there are only two parameters, $S_{\mathrm{wsp}} / S_{w}$ and $A_{\mathrm{nt}} / A$, which have the clear physical meanings, while models (Refs. 2-4) contain several empirical constants with no clear physical meanings. Particularly, the model (Ref. 5) presents totally four correlations in four saturation regions, each correlation 


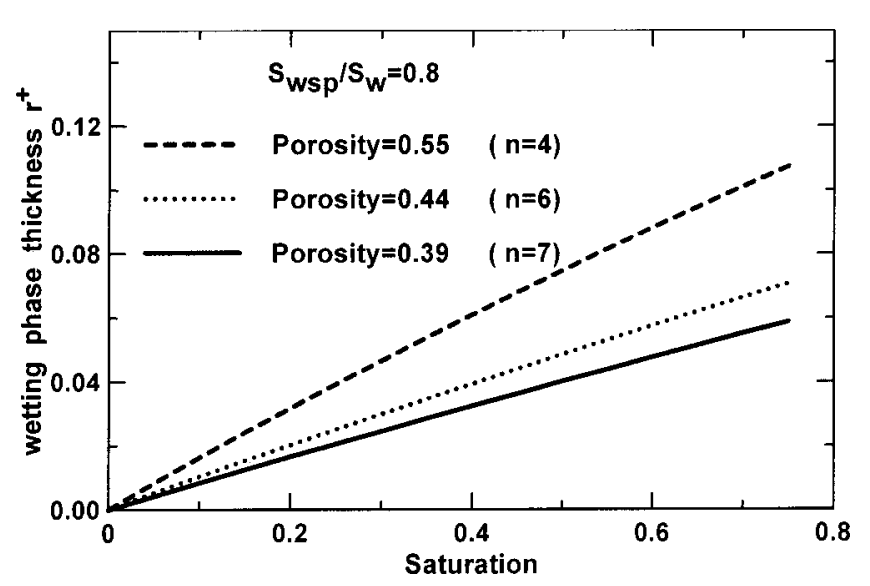

FIG. 4. Effect of the porosity on the wetting phase thickness $r^{+}$.

has two empirical constants. Therefore, the advantage of the present model over the other models is evident.

(b) Compared with numerical solutions such as the boundary element method (BEM), ${ }^{18}$ finite element method $(\mathrm{FEM}),{ }^{19}$ and finite difference method (FDM), ${ }^{20}$ the present analytical model also has the following advantages over the numerical solutions:

(1) The present model is analytically related to the several variables such as $\phi, \beta_{\mathrm{wg}}, \beta_{\mathrm{sg}}, S_{\mathrm{wsp}} / S_{w}, A_{\mathrm{nt}} / A$, while the numerical solutions cannot analytically provide such relationship.

(2) The solution of the present model is simpler and faster in calculation (less than one second in a microcomputer), and no inclusions are needed. While numerical solution by BEM requires many inclusions, this requires much computational time and needs a big computer memory.

(3) The accuracy by numerous methods such as the BEM, ${ }^{18}$ FEM, ${ }^{19}$ and FDM $^{20}$ usually depends on grid sizes (may change with different regions) and the number of iteration for convergence. But the present analytical solution does not have such a weakness. The results by Zou et $a .^{21}$ did indicate that the thermal-electrical analogy can give a better agreement with the experimental data than the FEM and FDM.

\section{RESULTS AND DISCUSSION}

In our previous work, ${ }^{13}$ we have justified that the constant fractal dimension $D_{f}=1.89$ can be approximately used to describe the porous medium if the porosity is in the range of $0.3-0.5$. If the porosity far deviates from this range, the Sierpínski carpets with different fractal dimensions may need to be constructed to simulate such porous media. Therefore, in this work we continue to use the Sierpínski carpet with the fractal dimension $D_{f}=1.89$ to model the three-phase/ unsaturated porous media.

Figure 4 compares the effect of the porosity (through stage $n$ ) on the wetting phase thickness $r^{+}=r / L^{(0)}$ in this model. It can be seen from the figure that the porosity has the significant influence on parameter $r^{+}$. The figure shows that the wetting phase thickness increases with the porosity. This can be interpreted as that when the porosity increases, the

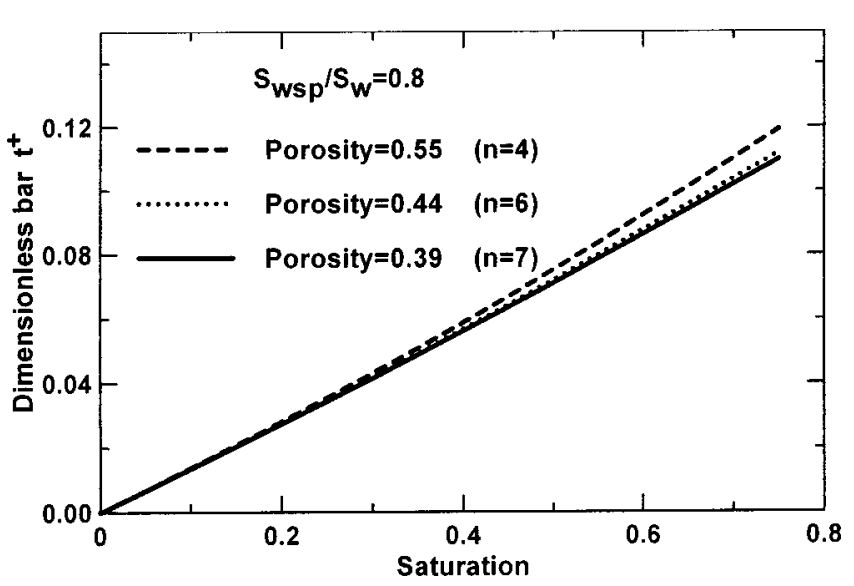

FIG. 5. Effect of porosity on the contact resistance $t^{+}$.

thickness of wetting phase surrounding particles increases too at a given saturation. The figure also indicates that the wetting phase thickness increases with saturation at a given porosity, this is consistent with physical situation.

Figure 5 compares the effect of the porosity on the contact parameter $t^{+}=t / L^{(0)}$. It is seen from the figure that the contact parameter $t^{+}$increases slightly with the porosity, but it increases linearly with saturation $S_{w}$. This is expected because when saturation increases, wetting phase content increases, leading to the increase of the contact parameter $t^{+}$.

Figure 6 depicts the effect of porosity on the thermal conductivity at different saturations. It is clear that the thermal conductivity increases as the porosity decreases when $\beta_{\mathrm{wg}}>1$ and $\beta_{\mathrm{sg}}>1$, see Fig. 6(a). This can be interpreted as that when the porosity decreases, the solid volume fraction with higher thermal conductivity increases, leading to the increase of the effective thermal conductivity. However, it is found that when $\beta_{\mathrm{wg}}<1$ and $\beta_{\mathrm{sg}}<1$, the effective thermal conductivity is slightly dependent upon porosity, see Fig. 6(b). But for $\beta_{\mathrm{wg}}<1$ and $\beta_{\mathrm{sg}}<1$, the opposite phenomenon is observed, i.e., the thermal conductivity increases with porosity. The reason is that when $\beta_{\mathrm{wg}}<1$ and $\beta_{\mathrm{sg}}<1$, the matrix space with higher thermal conductivity dominates the effective thermal conductivity, resulting in a higher effective thermal conductivity at a higher porosity.

Figure 7 compares the present model predictions at porosity of 0.44 [stage $n=6$, obtained by Eq. (2)] with the existing experimental data ${ }^{2}$ reported in Table I at porosity of 0.42. In the present model $A_{\mathrm{nt}} / A=0.55$ (the same value as that given by Ma et al. ${ }^{13}$ ), $S_{\mathrm{wsp}} / S_{w}=0.8$ (the same value as that given by Ref. 5) are chosen for best fitting the experimental data. Figure 7 also shows that the experimental thermal conductivities at porosity 0.42 are slightly higher than the present model predictions at porosity 0.44 . This is reasonable, see the discussion on Fig. 6(a) in the last sentence. In all, good agreement is found between the present model predictions and the existing experimental data.

Figure 8 is a comparison of the present model predictions at porosity of 0.55 [stage $n=4$, see Eq. (2)] with the experimental data ${ }^{2}$ reported in Table II at a porosity of 0.52 . The computations are also based on the parameters $A_{\mathrm{nt}} / A$ $=0.55$ and $S_{\mathrm{wsp}} / S_{w}=0.8$. From Fig. 8 , it is seen that good 


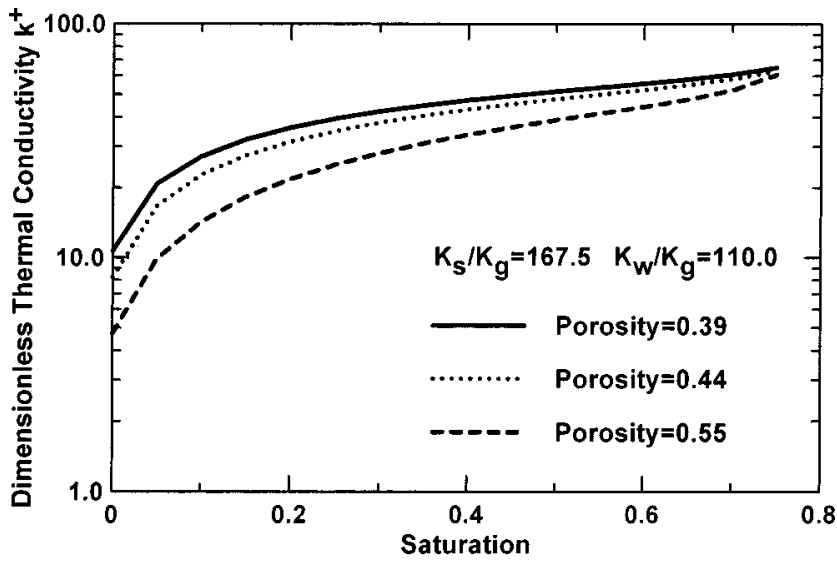

(a)

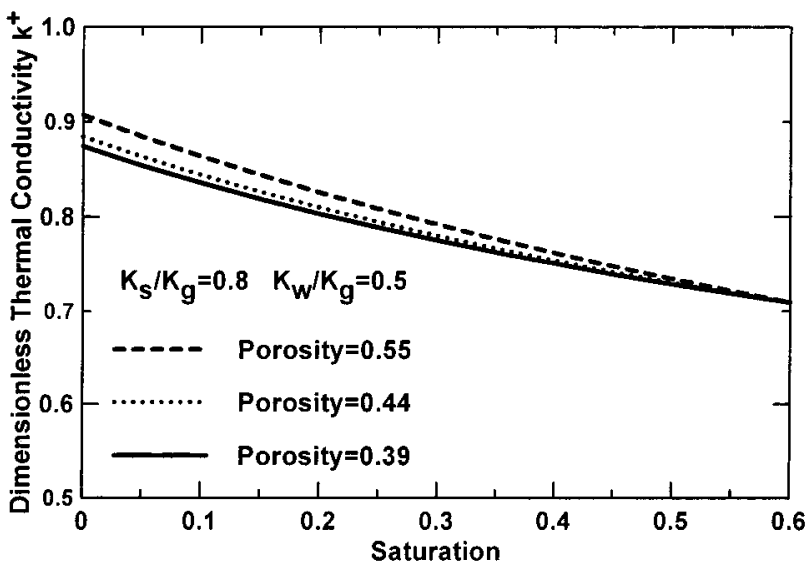

(b)

FIG. 6. Effect of porosity on the thermall conductivities at different ratios of $k_{s} / k_{g}$ and $k_{w} / k_{g}$.

agreement is again found between the present model predictions and experimental data. From Figs. 7 and 8, it can be seen that the accuracy of the present model is verified. Since no experimental data at $\beta_{\mathrm{wg}}<1$ and $\beta_{\mathrm{sg}}<1$ were reported in literature, the comparisons with the existing experimental data only at $\beta_{\mathrm{wg}}>1$ and $\beta_{\mathrm{sg}}>1$ are given in this work.

It should be noted that although the parameters $S_{\mathrm{wsp}} / S_{w}$ $(=0.8), A_{\mathrm{nt}} / A(=0.55)$ used in this model have the clear

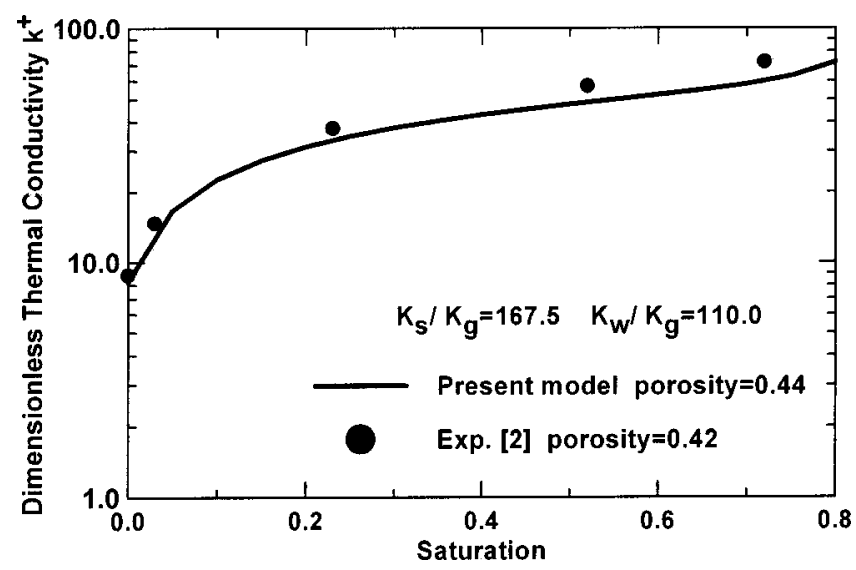

FIG. 7. A comparison between the present model predictions and experimental data at porosity $0.42-0.44$.
TABLE I. Experimental results, Dune sand [2]

\begin{tabular}{ccc}
\hline \hline Saturation $S_{w}$ & $k_{e}\left(\mathrm{Wm}^{-1} \mathrm{~K}^{-1}\right)$ & $k_{e}^{+}\left(\mathrm{Wm}^{-1} \mathrm{~K}^{-1}\right)$ \\
\hline 0.00 & 0.176 & 8.80 \\
0.03 & 0.294 & 14.70 \\
0.23 & 0.752 & 37.50 \\
0.52 & 1.137 & 56.85 \\
0.72 & 1.438 & 71.90 \\
\hline \hline
\end{tabular}

Note: The experimental conditions: Dune sand $k_{s}=3.35 \mathrm{Wm}^{-1} \mathrm{~K}^{-1}$, pore space $k_{g}=0.02 \mathrm{Wm}^{-1} \mathrm{~K}^{-1}$ and water $k_{w}=2.2 \mathrm{Wm}^{-1} \mathrm{~K}^{-1}$, at porosity $\varphi$ $=0.42$ temperature $20^{\circ} \mathrm{C}$, and here $k_{e}^{+}=k_{e} / k_{g}$.

physical meanings, their values are chosen by fitting the existing experimental data at porosities of 0.42 and 0.52 , therefore, they are approximate. These parameters may be related to porosity and should be further studied in a wider range of porosity (such as $0.2-0.8$ ). In this wider range of porosity, the Sierpínski carpets with different fractal dimensions may need to be constructed to simulate porous media. This might be our future work, while this paper presents our preliminary work.

\section{CONCLUSIONS}

An approximate model, a fractal geometry model, for the effective thermal conductivity of unsaturated porous media is derived based on the statistically self-similar characteristics of porous media and on the thermal-electrical analogy technique. The proposed thermal conductivity model is found to be a function of porosity (related to stage $n$ of Sierpínski carpet), the ratio of areas, the ratio of component thermal conductivities, and saturation, and every parameter in this model has clear physical meaning. The model predictions are compared with the existing experimental data, and the results show that the present model presents good agreement with the existing experimental data when $\beta_{\mathrm{wg}}>1$ and $\beta_{\mathrm{sg}}>1$. The recursive algorithm for the thermal conductivity by the proposed model is quite simple.

\section{ACKNOWLEDGMENT}

This work was supported by the National Nation Science Foundation of China through Project No. 10272052.

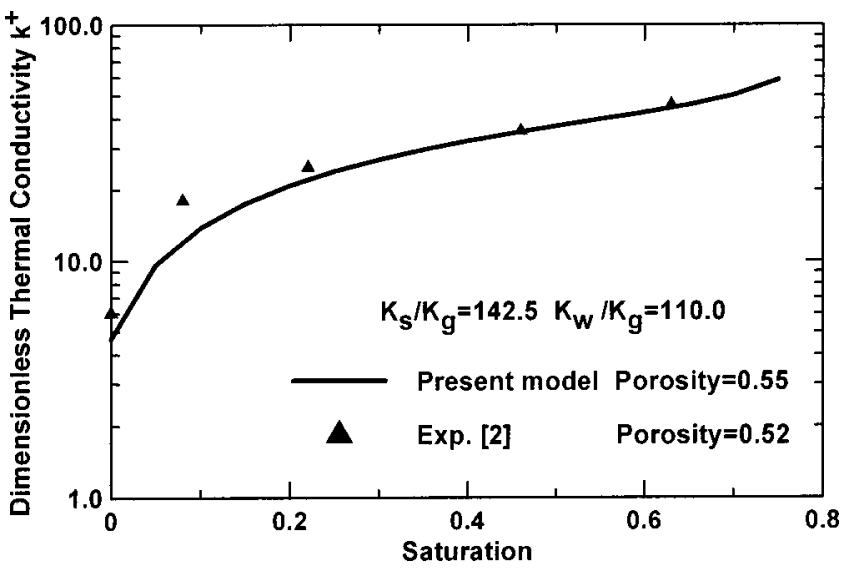

FIG. 8. A comparison between the present model predictions and experimental data at porosity $0.52-0.55$. 
TABLE II. Experimental results, Brick sand [2].

\begin{tabular}{ccc}
\hline \hline Saturation $S_{w}$ & $k_{e}\left(\mathrm{Wm}^{-1} \mathrm{~K}^{-1}\right)$ & $k_{e}^{+}\left(\mathrm{Wm}^{-1} \mathrm{~K}^{-1}\right)$ \\
\hline 0.00 & 0.12 & 6.0 \\
0.08 & 0.36 & 18 \\
0.22 & 0.50 & 24.9 \\
0.46 & 0.71 & 35.5 \\
0.63 & 0.92 & 45.8 \\
\hline
\end{tabular}

Note: The experimental conditions: Brick sand $k_{s}=2.85 \mathrm{Wm}^{-1} \mathrm{~K}^{-1}$, pore space $k_{g}=0.02 \mathrm{Wm}^{-1} \mathrm{~K}^{-1}$ and water $k_{w}=2.2 \mathrm{Wm}^{-1} \mathrm{~K}^{-1}$, at porosity $\varphi$ $=0.52$ and temperature $20^{\circ} \mathrm{C}$, here $k_{e}^{+}=k_{e} / k_{g}$.

\section{APPENDIX A}

For the 0 -stage carpet, the wetting phase content $S_{\text {wsp }}^{(0)}$ surrounding the particles can be obtained by

$$
\begin{aligned}
S_{\mathrm{wsp}}^{(0)}= & \frac{V_{\mathrm{wsp}}}{V_{p}}=\frac{V_{\mathrm{wsp}} / V_{t}}{V_{p} / V_{t}} \\
= & {\left[\left(\frac{\delta_{2}^{(0)}+\delta_{3}^{(0)}+\delta_{4}^{(0)}+\delta_{5}^{(0)}+\delta_{6}^{(0)}}{L^{(0)}}\right)^{2}\right.} \\
& \left.-\left(\frac{\delta_{3}^{(0)}+\delta_{4}^{(0)}+\delta_{5}^{(0)}}{L^{(0)}}\right)^{2}\right] / \varphi,
\end{aligned}
$$

where $L^{(0)}$ is the size of the 0 -stage Sierpínski carpet, $\varphi$ $=V_{p} / V_{t}, V_{t}=L^{(0)^{2}}$, and $\left(\delta_{3}^{(0)}+\delta_{4}^{(0)}+\delta_{5}^{(0)}\right) / L^{(0)}=1 / 3$. Set $\left(\delta_{2}^{(0)}+\delta_{6}^{(0)}\right) / L^{(0)}=r / L^{(0)}=r^{+}$, Eq. (A1) can be reduced to

$$
\begin{gathered}
S_{\mathrm{wsp}}^{(0)}=\left[\left(\frac{1}{3}+r^{+}\right)^{2}-\left(\frac{1}{3}\right)^{2}\right] / \varphi=\left[\left(\frac{1}{3}+r^{+}\right)^{2}-\left(\frac{1}{3}\right)^{2}\right] \\
/\left(\frac{8}{9}\right)=\frac{9}{8}\left[\left(\frac{1}{3}+r^{+}\right)^{2}-\left(\frac{1}{3}\right)^{2}\right] .
\end{gathered}
$$

Because of self-similarity, for the $n$-stage carpet, the wetting phase content $S_{\text {wsp }}^{(n)}$ surrounding the particles can be obtained by

$$
\begin{gathered}
S_{\mathrm{wsp}}^{(n)}=9\left[\left(\frac{9}{8}\right)^{n+1}-1\right]\left[\left(\frac{1}{3}+r^{+}\right)^{2}-\left(\frac{1}{3}\right)^{2}\right] \\
(n=0,1,2, \ldots,) .
\end{gathered}
$$

\section{APPENDIX B}

For the 0 -stage carpet, the barrier content $S_{\mathrm{wb}}^{(0)}$ of wetting phase can be obtained by

$$
S_{\mathrm{wb}}^{(0)}=\frac{V_{\mathrm{wb}}}{V_{p}}=4 \frac{\delta_{4}^{(0)}}{L^{(0)}} \times \frac{\delta_{1}^{(0)}}{L^{(0)}} / \varphi .
$$

Set $t^{+}=\delta_{4}^{(0)} / L^{(0)}=t / L^{(0)}$, dimensionless width of barrier. $\left(\delta_{2}^{(0)}+\delta_{6}^{(0)}\right) / L^{(0)}=r / L^{(0)}=r^{+}, \quad \delta_{1}^{(0)} / L^{(0)}=1 / 3-r^{+} / 2$ is dimensionless width of the first layer, and $r^{+} / 2$ is dimensionless thickness of wetting phase surrounding the particle in Fig. 2(a). Equation (B1) can then be reduced to

$$
S_{\mathrm{wb}}^{(0)}=\frac{V_{\mathrm{wb}}}{V_{p}}=4 t^{+}\left(\frac{1}{3}-\frac{r^{+}}{2}\right) /\left(\frac{8}{9}\right)=\frac{9}{8} \times 4 t^{+}\left(\frac{1}{3}-\frac{r^{+}}{2}\right) .
$$

Because of self-similarity, for the $n$-stage carpet, the barrier content $S_{\mathrm{wb}}^{(n)}$ of the wetting phase can be obtained by

$$
S_{\mathrm{wb}}^{(n)}=9\left[\left(\frac{9}{8}\right)^{n+1}-1\right] \times 4 t^{+}\left(\frac{1}{3}-\frac{r^{+}}{2}\right) \quad(n=0,1,2, \ldots,) .
$$

\section{APPENDIX C}

In Fig. 2(a), $\delta_{1}^{(0)}, \delta_{2}^{(0)}, \delta_{3}^{(0)}, \delta_{4}^{(0)}, \delta_{5}^{(0)}, \delta_{6}^{(0)}$, and $\delta_{7}^{(0)}$ represent the thicknesses of layers $1,2,3,4,5,6$, and 7 along the direction of heat flow through the 0-stage Sierpínski carpet. The thermal resistance $R_{1}^{(0)}$ of layer 1 is considered to be the sum of parallel resistances of blocks marked 11, 12, 13, and bar $t$, the resistance is

$$
\begin{aligned}
R_{1}^{(0)} & =\frac{R_{11}^{(0)} R_{12}^{(0)} R_{13}^{(0)}}{R_{12}^{(0)} R_{13}^{(0)}+R_{11}^{(0)} R_{13}^{(0)}+R_{11}^{(0)} R_{12}^{(0)}} \\
& =\frac{\delta_{1}^{(0)}}{L^{(0)} k_{g}\left[t^{+} \beta_{\mathrm{wg}}^{(0)}+\left(1-t^{+}\right)\right]},
\end{aligned}
$$

where $\beta_{\mathrm{wg}}^{(0)}=k_{w} / k_{g}$. Similarly, the thermal resistance $R_{2}^{(0)}$ of layer 2 is

$$
\begin{aligned}
R_{2}^{(0)} & =\frac{R_{21}^{(0)} R_{22}^{(0)} R_{23}^{(0)}}{R_{22}^{(0)} R_{23}^{(0)}+R_{21}^{(0)} R_{23}^{(0)}+R_{21}^{(0)} R_{22}^{(0)}} \\
& =\frac{\delta_{2}^{(0)}}{k_{g}\left[\left(l_{22}^{(0)}+r\right) \beta_{\mathrm{wg}}^{(0)}+\left(l_{21}^{(0)}+l_{23}^{(0)}-r\right)\right]} .
\end{aligned}
$$

The thermal resistance $R_{3}^{(0)}$ of layer 3 is

$$
R_{3}^{(0)}=\frac{\delta_{3}^{(0)} / k_{g}}{l_{31}^{(0)}+l_{33}^{(0)}-r+\beta_{\mathrm{wg}}^{(0)} r+\beta_{\mathrm{sg}}^{(0)} l_{32}^{(0)}} .
$$

The thermal resistance $R_{4}^{(0)}$ of layer 4 is

$$
R_{4}^{(0)}=\frac{\delta_{4}^{(0)} / k_{g}}{\left(l_{41}^{(0)}+l_{43}^{(0)}\right) \beta_{\mathrm{wg}}^{(0)}+\beta_{\mathrm{sg}}^{(0)} l_{42}^{(0)}} .
$$

Due to the symmetry, the thermal resistances $R_{5}^{(0)}, R_{6}^{(0)}$, and $R_{7}^{(0)}$ of layers 5,6 , and 7 can be found

$$
\begin{aligned}
& R_{5}^{(0)}=R_{3}^{(0)}, \\
& R_{6}^{(0)}=R_{2}^{(0)}, \\
& R_{7}^{(0)}=R_{1}^{(0)} .
\end{aligned}
$$

The total resistance of 0-stage Sierpínski carpet is considered as the series of layers $1,2,3,4,5,6$, and 7 , and it yields

$$
\begin{aligned}
R_{\mathrm{sc}}^{(0)}= & R_{1}^{(0)}+R_{2}^{(0)}+R_{3}^{(0)}+R_{4}^{(0)}+R_{5}^{(0)}+R_{6}^{(0)}+R_{7}^{(0)} \\
= & \frac{1}{k_{g}}\left\{\frac{\delta_{1}^{(0)}+\delta_{7}^{(0)}}{L^{(0)}\left[t^{+} \beta_{\mathrm{wg}}^{(0)}+\left(1-t^{+}\right)\right]}\right. \\
& +\frac{\delta_{2}^{(0)}+\delta_{6}^{(0)}}{\left(l_{22}^{(0)}+r\right) \beta_{\mathrm{wg}}^{(0)}+l_{21}^{(0)}+l_{23}^{(0)}-r} \\
& +\frac{\delta_{3}^{(0)}+\delta_{5}^{(0)}}{l_{31}^{(0)}+l_{33}^{(0)}-r+\beta_{\mathrm{wg}}^{(0)} r+\beta_{\mathrm{sg}}^{(0)} l_{32}^{(0)}}
\end{aligned}
$$




$$
\left.+\frac{\delta_{4}^{(0)}}{\left(l_{41}^{(0)}+l_{43}^{(0)}\right) \beta_{\mathrm{wg}}^{(0)}+\beta_{\mathrm{sg}}^{(0)} l_{42}^{(0)}}\right\} .
$$

\section{APPENDIX D}

Figure 3(a) gives a unit cell for nontouching particles in the unsaturated state. Figure 3(b) is the network of the thermal resistance of Fig. 3(a). In Fig. 3(a), the thermal resistance of gas (layers 1 and 5) of the length $\left(l_{t}-l_{s}-2 l_{w}\right)$ is

$$
R_{1}=\frac{1}{k_{g}} \frac{l_{t}-l_{s}-2 l_{w}}{l_{t}}=\frac{1}{k_{g}} \frac{\left(l_{t}-l_{s}-2 l_{w}\right) / l_{s}}{l_{t} / l_{s}} .
$$

The thermal resistance of gas-water (layers 2 and 4 ) of the length $\left(2 l_{w}\right)$ is

$$
R_{2}=\frac{2 l_{w}}{k_{g}\left(l_{t}-l_{s}\right)+k_{w} l_{s}}=\frac{1}{k_{g}} \frac{2 l_{w} / l_{s}}{\left(l_{t}-l_{s}\right) / l_{s}+\beta_{\mathrm{wg}}} .
$$

The thermal resistance of the gas-water-solid (layer 3 ) material of length $\left(l_{s}\right)$ is

$$
\begin{aligned}
R_{3} & =\frac{l_{s}}{k_{g}\left(l_{t}-l_{s}-2 l_{w}\right)+k_{w} 2 l_{w}+k_{s} l_{s}} \\
& =\frac{1}{k_{g}} \frac{1}{\left(l_{t}-l_{s}-2 l_{w}\right) / l_{s}+\beta_{\mathrm{wg}} 2 l_{w} / l_{s}+\beta_{\mathrm{sg}}} .
\end{aligned}
$$

Set $B=l_{t} / l_{s}=[1 /(1-\varphi)]^{1 / 2}$ (the same definition as that in Ref. 5) and $F=4 l_{w} / l_{s}=S_{w} \varphi /(1-\varphi)$ (the same definition as that in Ref. 5). The total resistance of Fig. 3(a) can be expressed as

$$
\begin{aligned}
R_{t}= & R_{1}+R_{2}+R_{3}=\frac{1}{k_{g}}\left(\frac{B-1-F / 2}{B}+\frac{F}{2\left[(B-1)+\beta_{\mathrm{wg}}\right]}\right. \\
& \left.+\frac{1}{\beta_{\mathrm{sg}}+F \beta_{\mathrm{wg}} / 2+(B-1-F / 2)}\right)
\end{aligned}
$$

So, dimensionless thermal conductivity for the nontouching particle portion is obtained as

$$
\begin{aligned}
k_{e, \mathrm{nt}}^{+}= & \frac{l_{t}}{R_{t} A_{t} k_{g}}=\left\{\frac{B-1-F / 2}{B}+\frac{F}{2\left[(B-1)+\beta_{\mathrm{wg}}\right]}\right. \\
& \left.+\frac{1}{\beta_{\mathrm{sg}}+F \beta_{\mathrm{wg}} / 2+(B-1-F / 2)}\right\}^{-1}
\end{aligned}
$$

where $A_{t}=l_{t} \times 1, \beta_{\mathrm{wg}}=k_{w} / k_{g}$ and $\beta_{\mathrm{sg}}=k_{s} / k_{g}$.

${ }^{1}$ I. Nozad, R. G. Carbonell, and S. Whitaker, Chem. Eng. Sci. 40, 857 (1985).

${ }^{2}$ A. K. Singh, R. Singh, and D. R. Chaudhary, J. Phys. D 23, 698 (1990).

${ }^{3}$ X.-J. Hu, J.-H. Du, S.-Y. Lei, and B.-X. Wang, Int. J. Heat Mass Transfer 44, 247 (2001).

${ }^{4}$ N. H. Abu-Hamdeh, A. I. Khdair, and R. C. Reeder, Int. J. Heat Mass Transfer 44, 1073 (2001).

${ }^{5}$ F. Gori and S. Corasaniti, J. Heat Transfer 124, 1001 (2002).

${ }^{6}$ A. J. Katz and A. H. Thompson, Phys. Rev. Lett. 54, 1325 (1985).

${ }^{7}$ J. F. Thovert, F. Wary, and P. M. Adler, J. Appl. Phys. 68, 3872 (1990).

${ }^{8}$ I. M. Young and J. W. Crawford, J. Soil Sci. 42, 187 (1991).

${ }^{9}$ P. M. Adler, J. Hydrol. 187, 195 (1996).

${ }^{10}$ J. M. Smidt and D. M. Monro, Fractals 6, 401 (1998).

${ }^{11}$ B. M. Yu and J. H. Li, Fractals 9, 365 (2001).

${ }^{12}$ C. Richter C and H. J. Viljoen, J. Appl. Phys. 93, 2663 (2003).

${ }^{13}$ Y. T. Ma, B. M. Yu, D. M. Zhang, and M. Q. Zou, J. Phys. D 36, 2157 (2003).

${ }^{14}$ B. B. Mandelbrot, The Fractural Geometry of Nature (W. H. Freeman, New York, 1982).

${ }^{15}$ J. E. Hutchinson, Indiana Univ. Math. J. 30, 713 (1981).

${ }^{16}$ J. Feder, Fractals (Plenum Press, New York, 1988).

${ }^{17}$ R. A. Crane and R. I. Vachon, Int. J. Heat Mass Transfer 20, 711 (1977).

${ }^{18}$ B. A. Baltz and M. S. Ingber, Eng. Anal. Boundary Elem. 19, 3 (1997).

${ }^{19}$ Md. R. Islam and A. Pramila, J. Compos. Mater. 33, 1699 (1999).

${ }^{20}$ B. W. James and G. S. Keen, High Temp. - High Press. 17, 477 (1985).

${ }^{21}$ M. Q. Zou, B. M. Yu, and D. M. Zhang, J. Phys. D 35, 1867 (2002). 